\title{
The effect of prunes on gastrointestinal health - a systematic review of randomised controlled trials
}

\author{
E Lever, J Cole, P Emery and K Whelan \\ Diabetes and Nutritional Sciences Division, King's College London, London SE1 9NH, UK
}

Chronic constipation affects $15-20 \%$ of people in the $\mathrm{UK}^{(1)}$. Lifestyle modifications such as increased dietary fibre intake are recommended as first line therapy but the evidence base for this is poor ${ }^{(2)}$. Prunes (dried plums) are commonly believed to have a laxative effect $^{(3)}$ and used as a traditional remedy to improve bowel function, however there has been no systematic review of the quality or number of clinical trials investigating the efficacy of prunes on gastrointestinal health in constipated or healthy subjects.

The aim of this study was to assess the effect of prunes on markers of gastrointestinal health in constipated and healthy subjects through a systematic review of randomised controlled trials (RCTs).

Sixteen electronic databases were searched, a hand search of reference lists and cited articles of relevant reports and review articles was performed and key opinion leaders were contacted. Only RCTs investigating the effect of prunes on gastrointestinal health were included. Two reviewers independently screened the relevant articles, extracted the data and assessed risk of bias.

Four RCTs were eligible ${ }^{(4-7)}$. All evaluated the effect of prune consumption versus another food on markers of gastrointestinal health. One study was conducted in constipated patients and three studies were in healthy subjects. Meta-analysis was not possible due to variability in how outcomes were assessed and reported. Due to lack of adequate reporting there was unclear risk of bias for many quality components. All four trials examined the effect of prunes on stool frequency, consistency and gastrointestinal symptoms. Only one, conducted in constipated patients, reported that prunes significantly increased stool frequency $(3.5 \pm 0.2 \mathrm{vs}$. $2.8 \pm 0.2$ complete spontaneous bowel movements/week) ${ }^{(6)}$. Two of the four studies reported that prunes led to an improvement in stool consistency (3.2 vs. 2.8 on 7-point Bristol stool scale $)^{(5,6)}$. One of the four studies examined the effect of prunes on stool weight and faecal bile acid concentration in which prunes significantly increased stool weight $(628 \mathrm{~g} \pm 43.9 \mathrm{vs} .514 \mathrm{~g}+/-34.072 \mathrm{hr}$ wet weight) and reduced faecal concentration of lithocholic acid $\left(0.95 \mathrm{mg} v s .1 .20 \mathrm{mg} / \mathrm{g}\right.$ dry weight stool) in healthy subjects ${ }^{(4)}$.

Despite the commonly held belief that prunes have a laxative effect, the evidence for this is limited. There was heterogeneity in methods and findings and studies were limited due to unclear risk of bias and lack of adequate reporting. It is unclear if the findings have clinical relevance. This review has highlighted the need for well conducted and reported RCTs investigating the effect of prunes on gastrointestinal health in constipated and healthy individuals.

The systematic review was made possible with funding from the California Dried Plum Board.

1. Suares NC and Ford AC (2011) Prevalence of, and Risk Factors for, Chronic Idiopathic Constipation in the Community: Systematic Review and Meta-analysis. Am J Gastroenterol.

2. Suares NC and Ford AC (2011) Systematic review: the effects of fibre in the management of chronic idiopathic constipation. 33, 895-901

3. Muller-Lissner SA, Kaatz V, Brandt W, Keller J and Layer P (2005) The perceived effect of various foods and beverages on stool consistency. Eur $J$ Gastroenterol Hepatol 17, 109-112.

4. Tinker LF, Schneeman BO, Davis PA, Gallaher DD and Waggoner CR (1991) Consumption of prunes as a source of dietary fiber in men with mild hypercholesterolemia. Am J Clin Nutr 53, 1259-1265.

5. Howarth, Petrisko Y, Furchner-Evanson A, Nemoseck T and Kern M (2010) Snack selection influences nutrient intake, triglycerides, and bowel habits of adult women: a pilot study. J Am Diet Assoc 110, 1322-1327.

6. Attaluri A Donahoe R, Valestin J, Brown K and Rao SS (2011) Randomised clinical trial: dried plums (prunes) vs. psyllium for constipation. Aliment Pharmacol Ther 822-828.

7. Lucas EA, Hammond LJ, Mocanu V, Arquitt AB, Trolinger A, Khalil DA, Smith BJ, Soung DY, Daggy BP and Arjmandi BH (2004) Daily consumption of dried plum by postmenopausal women does not cause undesirable changes in bowel function. Journal of Applied Research 4(1), 37-43. 